\title{
IgD, cyclooxygenase- 2 and ribosomal protein S6-PS240 immune response in a case of early psoriasis
}

\author{
Ana Maria Abreu Velez¹, Gabrielle M. Sabini², Michael S. Howard ${ }^{1}$ \\ ${ }^{1}$ Immunodermatology, Georgia Dermatopathology Associates Atlanta, Georgia, USA, ${ }^{2}$ Department of Dermatology, \\ North Atlanta Dermatology, Duluth, Georgia, USA
}

Corresponding author: Ana Maria Abreu Velez, E-mail: abreuvelez@yahoo.com

\begin{abstract}
Psoriasis is an inflammatory skin disease. Five classic types of psoriasis have been defined: plaque, inverse, pustular, guttate, and erythrodermic. The early psoriasis immunologic skin immune response is not well understood. Here we aim to show an immune and cell signaling response in a case of early psoriasis. A 56 year old female presented with a desquamative lesion on her right leg. A skin biopsy for hematoxylin and eosin (H\&E) and immunohistochemistry (IHC) staining was taken. The diagnosis indicated early psoriasis, and IHC showed positive IgD staining in the epidermal corneal layer, as well as positive staining with ribosomal protein S6-pS240 (RIBO) in the hyperproliferative epidermis. Cyclooxygenase-2 (COX-2) was also very positive in the granular layer in spots, at the basement membrane zone of the skin and in the inflammatory infiltrate in the dermis subjacent to hyperproliferative psoriatic areas. In an early case of psoriasis, we confirmed the presence of $\operatorname{IgD}, \mathrm{RIBO}$ and COX-2. Each molecule seems to be playing a role in inflammation and intracellular signaling pathways in early psoriasis. The role of $\operatorname{IgD}$ is unknown, and this case brings to light the complexity of the pathologic changes occurring in early psoriatic lesions.
\end{abstract}

Key words: Psoriasis; Cyclooxygenase-2; Ribosomal protein S6-ps240 phosphorylation site specific; IgD

\section{INTRODUCTION}

Psoriasis is a common, chronic inflammatory skin disease frequently presenting as well-demarcated, scaly plaques; many other clinical forms have been described [1]. The clinical presentation covers a spectrum including acute, chronic and relapsing forms; each stage seems to correlate with different immunologic markers. The chronic psoriasis immune response is characterized by hyperproliferative keratinocytes, infiltration of $\mathrm{T}$ cells, dendritic cells, macrophages and neutrophils, corneal ectopic expression of molecules and some autoreactivity [1-4]. In vivo binding of $\mathrm{IgG}$ in the stratum corneum of psoriatic lesions as well as in blood vessels has been previously documented [2,3] Autoantigen candidates for psoriasis include peroxiredoxin 2, ezrin, maspin, and heat shock protein 27. Some putative amino acid sequences with homologies to streptococcal proteins have been shown to react with the sera from psoriasis patients [5]. Multiple studies also point to possible alterations in the signaling pathways of nuclear factor$\kappa \mathrm{B}$, interferon- $\gamma$, and interleukins (IL)-23 and Il-17; and antigen presentation as central axes of psoriatic inflammation [1]. IL-17-producing dermal $\gamma \delta \mathrm{T}$ cells seem to be recently linked to psoriasis [4]. In our case of early psoriasis, we investigated the presence of cyclooxygenase-2 (COX-2), and ribosomal protein S6-ps240 (RIBO) [2,3]. A 56 year old female presented to the dermatologist for a scaling, itching plaque. The clinical examination revealed an erythematous, scaling plaque on the right leg. A skin biopsy for hematoxylin and eosin (H\&E) and immunohistochemical (IHC) staining was taken.

\section{CASE REPORT}

No patient identifiers were recorded, and our research was conducted following medical guidelines of

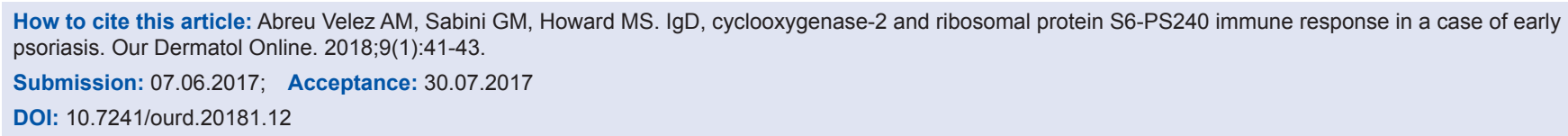


non-disclosure. Lesional skin was biopsied and studied utilizing hematoxylin and eosin (H\&E) staining, as well as via IHC. All techniques were performed as previously described $[2,3]$.

\section{IHC Double Staining}

Our double staining was performed utilizing a Leica (Buffalo Grove, IL, USA) double staining system. Specifically, for primary staining we utilized a Bond Max platform autostainer with bond polymer refined red detection DS9390, an alkaline phosphatase linker polymer and fast red chromogen (red staining). For our secondary staining, we utilized bond polymer refined detection DS9800, a horseradish peroxidase linker polymer and $\mathrm{DAB}$ chromogen (brown staining). Positive and negative controls were consistently performed. We utilized antibodies directed against ribosomal protein S6-pS240 phosphorylation site specific (RIBO), Clone DAK-S6-240; cyclooxygenase-2 (COX-2), and immunoglobulin D (IgD), all from Dako (Carpinteria, CA, USA).

\section{Microscopic Description}

The H\&E staining demonstrated focal confluent parakeratosis within the stratum corneum. In addition, scattered collections of neutrophils were seen within the stratum corneum. Overall, the epidermis displayed a mild psoriasiform hyperplasia. The stratum granulosum was focally attenuated. Minimal epidermal spongiosis was appreciated. Within the underlying dermis, a mild, perivascular infiltrate of lymphocytes, histiocytes and neutrophils was present (Fig. 1).

\section{IHC Staining}

Our IHC staining showed strong staining with COX-2, $\mathrm{IgD}$ and RIBO in the corneal layer. COX-2 and RIBO were very positive in cells around the inflamed dermal blood vessels under the epidermal lesions. The RIBO stain was most pronounced in the hyperproliferative regions of the epidermis (Fig. 1).

\section{DISCUSSION}

In this early case of psoriasis, we confirmed the presence of $\operatorname{IgD}, \mathrm{RIBO}$ and COX-2. Each molecule seems to be playing a role at this stage of psoriasis. $\mathrm{RIBO}$ is integral to protein translation, and is activated by phosphorylation via the mammalian rapamycin pathway [6]. We found expression of RIBO, showing differential phosphorylation in

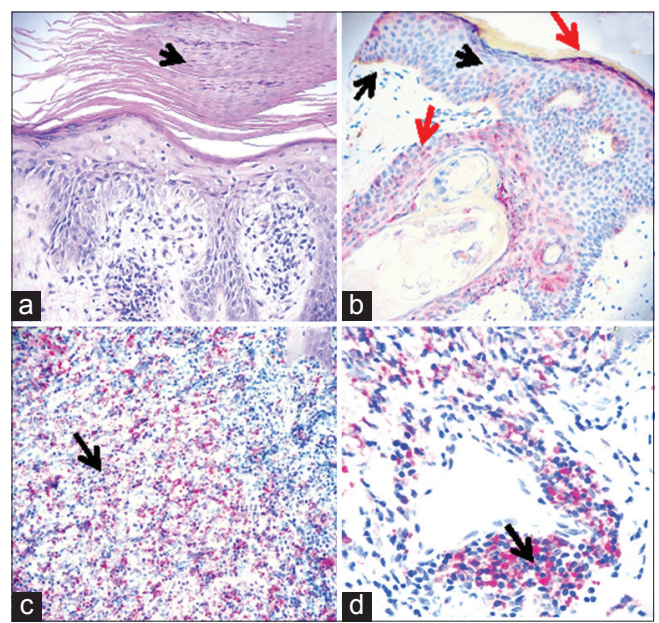

Figure 1: We highlight our main findings. (a) shows the psoriatic lesion using $\mathrm{H} \& \mathrm{E}$ staining with hyperkeratosis and Munro microabscesses (black arrow) (200X). (b) We present a double IHC stain with RIBO in red showing positivity in some spots in the corneal layer, as well as in the proliferative epidermis (red arrows) (200X). COX-2 is positive in different areas, mainly in discrete granular layer spots and at the basement membrane zone of the skin (black arrows, punctate brown staining). (c) Strong expression of COX-2 in the inflammatory infiltrate under a epidermal psoriatic lesion (red staining; black arrow)(100X) and a few cells staining positive for RIBO in brown. (d) A strong expression of COX-2 in the inflammatory infiltrate around dermal blood vessels (red staining; black arrow) (400X).

distinct epidermal layers; the expression was most pronounced in hyperproliferative regions. Based on our previous [2,3], and current findings and those of others, differential S6 phosphorylation may play a role in abnormal keratinocyte proliferation/differentiation in psoriasis, although only a few cases have been documented [2,3,5]. A larger series of cases is needed to adequately study the role of RIBO in intracellular pathways in psoriasis patients.

In regard to COX-2, we have previously shown positivity of this molecule in psoriatic skin and associated with inflammatory cells. Our findings here confirm our previous findings; however, the previous findings were in chronic cases $[2,3]$. Other authors in a recent study compared normal skin versus psoriatic skin; they compared the expression of both COX-2 and RIBO before and after treatment (using salt water baths and artificial ultraviolet (UVB) radiation, common therapeutic options for psoriasis) [6]. These authors studied the untreated versus the treated samples by testing COX-2 mRNA; this molecule was significantly increased in UVB irradiated normal psoriatic epidermis models versus normal skin [6].

Concerning the positivity of $\operatorname{IgD}$, one previous study's findings are relevant to our present study. Other authors had studied the mean values of serum immunoglobulins 
A, G, M and D levels in 42 psoriasis patients and controls, showing no differences between the groups [7]. They also searched for serum antiglobulin antibodies, yielding negative results; an antinuclear factor could be demonstrated in only $4.8 \%$ of the cases [7]. The authors suggested that serum antiglobulins became exhausted within the lesions; this hypothesis is supported by our preliminary findings of focal deposition in the stratum corneum of IgG, IgM and complement. Consulting the online PUBMED database, we could not found other studies linking IgD and psoriatic lesions.

In conclusion, our case of early psoriasis shows expression of IgD, RIBO and COX-2. Larger studies are needed to confirm any pathologic association between psoriasis and these molecules.

\section{ACKNOWLEDGEMENT}

Jonathan S. Jones, HT (ASCP) at Georgia Dermatopathology Associates provided excellent technical assistance.

\section{Abbreviations}

Hematoxylin and eosin (H\&E), immunohistochemistry (IHC), cyclooxygenase-2 (COX-2), ribosomal protein S6-pS240 phosphorylation site specific (RIBO).

\section{REFERENCES}

1. Boehncke WH. Etiology and Pathogenesis of Psoriasis. Rheum Dis Clin North Am. 2015;41:665-75.

2. Abreu-Velez AM, Googe PB, Howard MS. Immune reactivity in psoriatic Munro-Saboureau microabscesses, stratum corneum and blood vessels. N Am J Med Sci. 2012;4:257-65.

3. Abreu-Velez AM, Googe PB, Jr, Howard MS. Ribosomal Protein S6-pS240 is expressed in lesional skin from patients with autoimmune skin blistering diseases. N Am J Med Sci. 2013;5:604-8.

4. Cai Y, Fleming C, Jun Yan J. New insights of T cells in the pathogenesis of psoriasis. Cell Mol Immunol. 2012;9:302-9.

5. Besgen P, Trommler P, Vollmer S, Prinz JC. Ezrin, maspin, peroxiredoxin 2, and heat shock protein 27: potential targets of a streptococcal-induced autoimmune response in psoriasis. J Immunol. 2010;184:5392-402.

6. Ruf MT, Andreoli A, Itin P, Pluschke G, Schmid P. Ribosomal protein S6 is hyperactivated and differentially phosphorylated in epidermal lesions of patients with psoriasis and atopic dermatitis. Br J Dermatol. 201;171:1533-6.

7. Gambichler T, Terras S, Skrygan M. Cyclooxygenase 2 expression and apoptosis in normal and psoriatic epidermis models exposed to salt water soaks and narrowband ultraviolet B radiation. J Eur Acad Dermatol Venereol. 2015;29:180-83.

8. Nickoloff BJ. Skin innate immune system in psoriasis: friend or foe? J Clin Invest. 1999;104:1161-4.

Copyright by Ana Maria Abreu Velez, et al. This is an open-access article distributed under the terms of the Creative Commons Attribution License, which permits unrestricted use, distribution, and reproduction in any medium, provided the original author and source are credited.

Source of Support: Nil, Conflict of Interest: None declared. 